\title{
Las políticas públicas en la gestión del agua subterránea y su incidencia en el desarrollo rural.
}

Public policies in groundwater management and their impact on rural development.

Ec. Marjorie Ordóñez,

Mgs, Gricelda América Herrerra,

Dr. Wayky Luy Navarrete,

Dr. Jesús Merino Velásquez

Fecha de recepción: 4 de noviembre de 2018

Fecha de aceptación: 20 de diciembre de 2018 


\title{
Las políticas públicas en la gestión del agua subterránea y su incidencia en el desarrollo rural.
}

\author{
Public policies in groundwater management and their impact on rural \\ development.
}

\author{
Marjorie Ordóñez ${ }^{1}$, Gricelda América Herrerra ${ }^{2}$, Wayky Luy Navarrete ${ }^{3}$, Jesús Merino \\ Velásquez ${ }^{4}$
}

Como citar: Ordoñez, M., América, G., Luy, W., Merino, J., (2019). Las políticas públicas en la gestión del agua subterránea y su incidencia en el desarrollo rural, Revista Universidad de Guayaquil. 128(1), 1-11. DOI: https://doi.org/10.53591/rug.v128i1.1374

\section{RESUMEN}

El presente artículo tiene como objetivo analizar la importancia que tiene las políticas públicas para el desarrollo rural y el rol de la administración gubernamental de turno de garantizar que se cumplan los derechos humanos, siendo uno de los derechos vitales el acceso a agua limpia y el tratamiento de los desechos sólidos, en el desarrollo social y económico de una parroquia con características únicas dentro del contexto ambiental. Este estudio es cualitativo, de características descriptivas, es documental con revisión de los autores más relevantes de la materia relacionando los aspectos en común que cada uno de ellos considera básico en el análisis de las políticas públicas. El resultado de la investig ación revela que el desarrollo social y económico de una parroquia rural está estrechamente vinculado al cumplimiento de las políticas públicas congruentes a la visión de un estado haciendo que las mismas tengan participación de la comunidad, así como de los organismos seccionales gubernamentales y la actuación privada de organismos internacionales dedicadas a prestar su asesoramiento técnico.

Palabras clave: Política pública; desarrollo rural, participación comunitaria

\begin{abstract}
This article aims to analyze the importance of public policies for rural development and the role of the government administration on duty to guarantee that human rights are fulfilled, one of the vital rights being access to clean water and treatment of solid waste, in the social and economic development of a parish with unique characteristics within the environmental context. This study is qualitative, with descriptive characteristics, it is documentary with a review of the most relevant authors on the subject, relating the common aspects that each of them considers basic in the analysis of public policies. The result of the investigation reveals that the social and economic development of a rural parish is closely linked to the fulfillment of public policies consistent with the vision of a state, making them have the participation of the community, as well as government sectional agencies and the private action of international organizations dedicated to providing technical advice.
\end{abstract}

Keywords: Public politics; rural development, community participation

\footnotetext{
${ }^{1}$ Investiga dora Independiente. Correo electrónico: maitog1978@gmail.com

${ }^{2}$ Doctora en Ingeniería Geográfica Universidad Península de Santa Elena.Correo electrónico: griceldaherrer@gmail.com

${ }^{3}$ Doctor en Administración de la Educación Universidad Nacionalde Tumbes. Correo electrónico: wluyn@ untumbes.edu.pe

${ }^{4}$ Doctor en Administración Universidad Nacional de Tumbes. Correo electrónico: jmerinov@untumbes.edu.pe
} 


\section{INTRODUCCIÓN}

En el Ecuador, país democrático, todos sus habitantes gozan de deberes como de derechos, por lo cual el Estado tiene como deber primordial garantizar sin discriminación alguna el goce a todos sus ciudadanos de los derechos establecidos tanto en la Constitución, que es la Norma Suprema, así como los establecidos en los instrumentos internacionales, derechos tales como; la salud, la alimentación, la seguridad social y el agua. Por lo cual, para garantizar que se cumplan todos estos derechos a sus habitantes, el gobierno de turno traza su línea de acción, planificando a través de proyectos, programas y actividades, que pretende realizar a través de sus Planes de Gobierno, el mismo que tiene una duración de cinco años, el mismo tiempo que estaría en su mandato constitucional. Paralelamente, como uno de los derechos que tienen los habitantes de un país, se encuentra el derecho al acceso a agua potable que haya sido tratada de forma segura y que se disponga de un tratamiento efectivo de los residuos sólidos.

Para esto, el Estado dispone de instrumentos que le permitan efectivizar que se cumplan los derechos humanos y con esto garantizar una vida digna. Los instrumentos de los que se está haciendo relación se las conoce como las políticas públicas, tal como las define Muller (2007) que constituyen el modo como se le da congruencia y sostenibilidad a la toma de decisiones y a la gestión que hacen los gobiernos. Es por esto que la ciencia política y las políticas públicas comparten temas concernientes al sistema, régimen político, la fortaleza institucional, la gobernabilidad, el poder legislativo, el poder judicial y que junto a la sociedad civil comparten.

Por otra parte, el recurso agua, llamada también líquido vital, otras veces denominada como oro líquido es de importancia porque además de ser indispensable para el desarrollo y existencia del ser humano, para la biodiversidad y el medio ambiente, también permite llevar a cabo muchas actividades como la agricultura, la industria, además de ser una fuente generadora de energía eléctrica, garantiza el bienestar y la seguridad alimentaria y con esto el desarrollo social y económico de los habitantes.

El objeto de estudio de esta investigación es la parroquia de Manglaralto, una población rural ubicada en la provincia de Santa Elena, la misma que se encuentra en el oeste del Ecuador, está conformada políticamente por los cantones de Santa Elena, La Libertad y Salinas y estos cantones a su vez están conformadas por parroquias. El cantón Santa Elena está integrado por las parroquias rurales de Chanduy, Atahualpa, Simón Bolívar, Colonche, Manglaralto y San José de Ancón y por las parroquias urbanas de Ballenita y Santa Elena, todo este cantón comprende 60 comunas y 137 recintos.

La parroquia de Manglaralto, al ser una comunidad rural, tiene características tanto de una ciudad pequeña así como al mismo tiempo goza de una privilegiada riqueza natural y esto se puede describir en los dos sectores marcados que posee, primero una franja costera, que goza de unas privilegiadas playas y hacia dentro se encuentra una espesa y hermosa vegetación que lo hace único y por esto lo denominaron Bosque Protector de Chongón-Colonche, con características únicas de tipo campestre, rural, declarado como Bosque Protector en el año 1994, ocupa cerca del 40\% del territorio de la parroquia, motivo por el cual sus pobladores se han dedicado a actividades económicas relacionadas al ecoturismo y la conservación ambiental. La cabecera Parroquial es Manglaralto, tiene una población de 29512 habitantes, datos según el Censo de Población y de Vivienda, (INEC, 2010).

La parroquia de Manglaralto está conformada por 18 comunas, estas organizaciones sociales es una forma ancestral de organización territorial, que tienen una participación muy activa en la zona rural y con un nivel importante de convocatoria. Por otro lado, la cuenca del río Manglaralto está ubicada en la parte central de la parroquia Manglaralto, esta cuenca hidrográfica limita al norte con la cuenca del río Olón (Ayampe) y al sur con la cuenca del río Valdivia, constituye la principal fuente de alimentación del acuífero de Manglaralto. 
Por iniciativa y gestión propia de las comunas fueron creadas las juntas de agua potable, que son organizaciones comunitarias rurales creadas para solucionar problemas de abastecimiento de agua. La Junta Administradora de Agua Potable Regional de Manglaralto (JAAPMAN), provee del servicio de agua a las comunas de Montañita, Manglaralto, Cadeate, San Antonio, Río Chico y Libertador Bolívar. Cabe indicar que esta Junta de Agua, ha proporcionado de agua por medio de 12 pozos perforados a los habitantes de estas comunas, el consumo propio de los pobladores más el consumo de una población flotante, que se genera por ser reconocido como un destino turístico, ha hecho que la demanda sea creciente. Por otra parte, cabe indicar que este abastecimiento se encuentra en riesgo por los diferentes problemas que se han venido presentando; como por ejemplo la sobreexplotación del acuífero, las pocas precipitaciones para la recarga natural y la contaminación por intrusión salina.

\section{El agua como un derecho}

Dentro de los derechos que tienen los seres humanos, está el derecho al acceso a agua potable y al saneamiento. Este derecho se encuentra reconocido por la Asamblea General de las Naciones Unidas, en julio del 2010, que declara el derecho a tener acceso a una cantidad de agua suficiente para el uso doméstico y personal, entre 50 y 100 litros de agua por persona y día, agua segura, aceptable y asequible. El costo del agua no debería superar el 3\% de los ingresos del hogar y debe ser accesible físicamente, la fuente debe estar a menos de 1.000 metros del hogar y su recogida no debería superar los 30 minutos. (ONU, 2010).

Sin embargo, a pesar de ser reconocido como derecho, los problemas por acceder a este recurso vital aún persisten y Ecuador no es la excepción. La ONU sostiene que existen miles de millones de personas, principalmente en áreas rurales, que aún carecen de estos servicios básicos. En todo el mundo, una de cada tres personas no tiene acceso a agua potable salubre, dos de cada cinco personas no disponen de una instalación básica destinada a lavarse las manos con agua y jabón, y más de 673 millones de personas aún defecan al aire libre.

El acceso al agua como un derecho, inicia su recorrido a partir del marco de la Conferencia de las Naciones Unidas sobre el Agua en el año 1977, la cual reconoció que todos los pueblos "tienen derecho a acceder al agua potable en cantidad y calidad iguales para las necesidades esenciales de todos", independientemente del nivel de desarrollo económico alcanzado. Años después, en noviembre de 2002, el Comité de Derechos Económicos, Sociales y Culturales adoptó la Observación General núm. 15 sobre el derecho al agua, y en el artículo I.1 establece que "El derecho humano al agua es indispensable para una vida humana digna", también define el derecho al agua como el derecho de cada uno a disponer de agua suficiente, saludable, aceptable, físicamente accesible y asequible para su uso personal y doméstico.

La Observación General núm. 15 de la ONU creada en el 2010 y el Pacto Internacional sobre Derechos Económicos y Sociales y Culturales (PIDESC) constituyen el documento oficial que establecen que todos los Estados deberán asegurar que todas las personas puedan disponer de agua suficiente, salubre, aceptable, accesible y asequible para el uso personal y doméstico. Dado esto, recae en el Estado considerar una gobernabilidad, la cual se relaciona a la generación de disposiciones legales, políticas e institucionales, y a comportamientos y mecanismos, que sean suficientemente estables y eficientes; un financiamiento adecuado, centrado en la sostenibilidad del servicio; y, la existencia de políticas, los marcos legales y regulatorios, financiamiento, participación de la población, dentro de un entorno adecuado, que permitan el reconocimiento del derecho al agua potable (BID \& ONU-HABITAT, 2011).

Domínguez (2010) sostiene que los Estados deben cumplir, hacer cumplir y respetar el derecho al agua, esto implica garantizar la no discriminación al acceso, un trato equitativo y respetar sus usos tradicionales; e, impulsar la participación de otros actores cuando no se provea este recurso, por lo 
que el reconocimiento de este derecho provee de mecanismos legales o políticos para empoderar a la ciudadanía por lo que la solución es una cuestión de empoderamiento social y actuación jurídica.

Todo este marco regulatorio no ha sido suficiente para que todos tengan acceso al agua, este déficit de cobertura del servicio de agua conlleva a cuestionar la capacidad institucional y de gestión del Estado.

\section{Parroquia rural de Manglaralto}

Manglaralto es una parroquia rural, ubicada al norte de la provincia de Santa Elena, cuenta con una extensión de 497,4 km², en la costa del Ecuador, goza de una belleza natural extraordinaria, debido a su ubicación estratégica, que la hace privilegiada ya que posee al mismo tiempo de un borde costero, con hermosas playas y adentrándose se puede apreciar con el majestuoso Bosque Protector de Chongón-Colonche, el mismo que ocupa el $40 \%$ del territorio de la parroquia. Por todas estas bondades de la naturaleza que goza esta parroquia rural es motivo para que su gente se dedique a actividades ecoturísticas, agrícolas y al mismo tiempo preservando su medio ambiente, la cabecera Parroquial es Manglaralto y se encuentra a 5 minutos de Montañita.

Según datos proporcionados por el INEC, Censo Poblacional y de Vivienda (2010), su población es de 29.512 habitantes, esta delimita al norte con Manabí, al sur con la Parroquia Colonche, al este con el cantón Jipijapa, de la provincia de Manabí y al oeste con el Océano Pacífico.

La parroquia de Manglaralto está conformada políticamente por seis comunas como son Montañita, Manglaralto, Cadeate, San Antonio, Río Chico, Libertador-Bolívar. Por lo tanto, la Junta de Agua Potable Regional Manglaralto, administrada por seis representantes de cada una de las comunidades rurales, tiene como misión abastecer de agua a todas estas comunas, actualmente suministra de agua a 23.586 habitantes

Las organizaciones Comunales son organismos reconocidos legalmente por la Constitución de la República del Ecuador, su forma de gestionar y actual lleva muchísimos años atrás y es por esto que fue reconocida como una forma ancestral de organización territorial. Todas estas organizaciones a su vez están agrupadas en la Federación de Comunas Provincial de Santa Elena. Por otra parte, es importante señalar que su actuación es muy notable e influyente en las zonas rurales, tienen poder de convocar y participar activamente en conjunto con los Gobiernos Autónomos Descentralizados.

$\mathrm{Al}$ momento existen 18 comunas en el territorio parroquial, constituidas legalmente y amparadas en la Ley de Comunas de 1938 y reguladas por el Ministerio de Agricultura y Ganadería (MAGAP), y estas comunas a su vez están agrupadas y representadas por la Federación de Comunas de la provincia de Santa Elena, las mismas que son organizaciones sociales que representan a los territorios de la zona rural del cantón. Las comunas que integran la parroquia de Manglaralto, son: San José, San Antonio, San Francisco de las Núñez, Barcelona, Curia, Cadeate, Dos mangas, Atravesado, La entrada, Montañita, Olón, San Pedro, Sinchal, Sitio Nuevo, Valdivia, Pajiza, Río Chico, Río Blanco, San Vicente de Loja y La Rinconada.

\section{Políticas públicas}

Las políticas públicas o ciencias de las políticas, tiene su origen en la segunda mitad del siglo XX, en Estados Unidos. Harold Lasswell fue el propulsor y pionero, quien se desempeñó como docente e investigador en ciencia política y luego incorporó estos conocimientos científicos al manejo del gobierno de ese país, sus valiosos trabajos se enfocaron en optimizar la gestión administrativo, así como la gestión que venía desempeñando el gobierno del Estado, por medio de una nueva norma o método que sin lugar a dudas la llamaría las ciencias de las políticas (Policy Sciences). 
Según Nelson (2001), las politicas públicas nacen debido a la unión de algunas situaciones particulares, las que se destaca el régimen de un gobierno demócrata estable, que predomino la creación de organismos que se dedicaban exclusivamente a la investigación y a brindar asesoría de forma independiente, todo este renacer de organizaciones, condujo al gobierno de Estados Unidos a la planificación, creación e implementación de una gran cantidad de programas sociales en educación, salud y servicios públicos, estos programas lo condujeron a llamarse "Welfare State", Estado de Bienestar. Así mismo, otro factor determinante fue el filosófico y al continuar con la ideología de quien fue presidente de Estados Unidos, Woodrow Wilson, quien gozaba de excelsos conocimientos y una preparación académica única, pretendio hacer cambios en la administración pública, buscó potenciar las capacidades tanto administrativas y profesionales del Estado moderno, era indispensable para el país, así como para la ciencia política, lo que estuvo acompañado de una gran confianza social en la capacidad técnica para resolver problemas de la sociedad.

Consecutivamente, Fernandez (1996) señala que los presidentes de Estados Unidos, John F. Kennedy y Lyndon B. Johnson, impulsaron notablemente el desarrollo del estudio de las políticas. La imagen difundida fue que desde la ciencia de conocimiento pragmático mejoraría el desempeño de la administración y la gestión gubernamental del Estado, propiciando un "buen gobierno" o un "gobierno ilustrado". Por lo que, bajo este contexto, surgió la propuesta de Harold Lasswell, quien, junto con Daniel Lerner, crean las denominadas Policy Sciences (Ciencias de las Políticas en 1951, poniendo a la ciencia al servicio de la humanidad. (Nelson, 2001).

Harold Laswell, escribe sus dos magníficas obras, la primera titulada La orientación hacia las políticas en 1951 y tuvo que transcurrir veinte años para su segunda obra que la llamó, La concepción emergente de las ciencias de política en 1971, toda su obra se fundamenta en la concepción de un trabajo multi-inter-disciplinario, que pretende conocer y exponer como se forman y ejecutan las llamadas políticas, buscando la mejora de su contenido, con conocimiento utilizable, ya que era menester de la época el uso de conocimientos científicos y tecnológicos para atender los problemas sociales y de un gobierno, enfocado en la toma de decisiones.

Por esta razón, Lindblom \& Cohen (1979) manifestaron que este sería un trabajo transdisciplinario alrededor del resultado y el proceso de las políticas públicas, y tenga como fin el conocimiento y la explicación en cómo se forman y ejecutan las políticas, mejorando su contenido con la interacción del conocimiento usable.

Por otro lado, Aguilar (1996) esquematiza a la policy sciences en esa responsabilidad primordial y transcendental de la libertad y la democracia, el respeto y el compromiso netamente humanista que siempre habrá cosas que mejorar y paralelamente el uso de la metodología científica que permita estudiar e intervenir en la política.

Lasswell (1996) asevera que el que investiga las políticas debe enfocarse en indagar y recoger un conjunto de competencias científicas que le permitan contribuir a la creación de una teoría y una destreza fiable para la solución de problemas todo esto en el contexto del interés público. Paralelo a esto, así mismo el aporte de Losada (2003) indica que el hacedor del análisis y creador de las políticas, es primero un aclarador, motivado y comprometido con el mejoramiento, la educación y la ilustración de lo público, comprometido con la disminución de la coacción del poderío de los burócratas y oligarcas. Para Torgenson, avivar los ambientes de libertad y no dominar con el control, es el único fin que persigue este arte y carrera de las políticas. (1996).

Garson (1996), señala que la contribución que hace Laswell sobre las ciencias de las políticas va más allá, ya que esta ciencia se nutre y recoge cada una de las metodologías científicas de todas las doctrinas que la conforman, construyendo un estudio científico de la realidad, de y en las políticas, lo que equivale que va más allá de las características pragmático y humano. 
Así mismo, Laswell (1996) indica que el estilo estatal, la intervención cívica deberían ser otros materiales que maneje la ciencia de políticas, en vista que sus resultados tienen, ya que sus resultados tienen existencia jurídica, como son la observación de los hechos, la experiencia, con una serie de elementos relacionados entre sí y formal.

Las políticas públicas, como una subdisciplina de la ciencia políticas, como lo manifiesta Goodin y Klingemann (2001), empezó a nombrárselas en espacios estatales y de la academia en países como España, Francia y Estados Unidos y así mismo en agencias multilaterales y de desarrollo, Banco Mundial (BM) y el Banco Interamericano de Desarrollo(BID).

Mientras tanto, el interés que tiene la ciencia política por el estudio de las políticas públicas, es porque el Estado y su administración, reflejan la interconexión con un conjunto de instituciones que toman decisiones para resolver tantos problemas y desajustes sociales. Según Muller (2007), la forma como se le da coherencia, integralidad y sostenibilidad a las decisiones y acciones de los gobiernos es a lo que se dedican las políticas públicas, precisamente porque la ciencia política y las políticas públicas les conciernen temas de gobernabilidad, sistema, régimen político, el poder judicial, la fortaleza institucional, la gobernabilidad, el poder legislativo, y la sociedad civil; es así que las políticas públicas forman parte de lo que es la política, dando paso al convertirse esta en el tema de estudio de la ciencia política.

Se puede conceptualizar a las políticas públicas como el accionar directo o a través de intermediadores de las instituciones estatales, con el fin de influenciar en mejoras a la vida de los civiles. Chandler y Plano, señalan que en vista que la sociedad presenta sus demandas, la acción gubernamental propicia responder acertadamente con una solución, para esto el gobierno, hace uso de los recursos de manera estratégica y propicia menguar el peso de los problemas de toda una sociedad.

Es por esta razón que Pallares, propone que para el análisis efectivas de las Políticas Públicas que proponga el ente estatal, se debe iniciar planteando tres grandes interrogantes, sobre que políticas lleva a cabo el Estado en sus diferentes escenarios, cómo las elabora y finalmente como las evalúa y según sea el caso, cómo las va a cambiar, estas interrogantes en marcha son algunas pautas en el estudio de las Políticas Públicas.

En la década de 1960 en América Latina, se dieron los primeros estudios y aportes de las políticas públicas, en conjunto con todos los cambios que atravesaron el Estado y la sociedad, en lo que concierne a lo político y económico. Roth (2002 y 2006), Muller(1998 y 2006) y Zimerman (2001) exponen como ha ido evolucionando el tema del análisis de las políticas públicas desde la década del sesenta, en América Latina, el tema central se centró en la elaboración de estudios sobre cómo se dio la implementación de las decisiones y las dificultades del modelo llamo "Estado Bienestar", según transcurrían los años, en la década de los setenta, cobro importancia el análisis comparando la aplicación del modelo de Estado de Bienestar y lo que se venía desarrollando en América Latina, donde se pone en cuestionamiento la efectividad del Estado para formular políticas públicas efectivas, ya en los años ochenta, los estudios se centran en la conceptualización de lo que son y para qué sirven las agendas gubernamentales y el papel de los distintos actores que van influir en el quehacer de las políticas públicas, teniendo como consecuencia, grandes debates políticos y sociales, impulsando un cambio en el modelo de desarrollo y el papel del Estado, como el tamaño, el criterio de pertinencia, y las funciones, por lo que se dio una gran reforma al estudio de las políticas públicas, para la década del noventa, se dio una transformación histórica que marco esta década como fue el giro que se dio desde un modelo de democracia representativa hacia uno de democracia participativa, por lo cual los ciudadanos tienen voz y voto en las decisiones públicas y el papel del Estado, recobra importancia, al convertirse en el receptor de las demandas de los ciudadanos y que ellos mismos presenten las soluciones, logrando la participación de la ciudadanía en las decisiones públicas.

Es así que se marca la transformación de Estados liberales de corte neoclásico, con una gestión público-administrativa eficiente, donde los recursos y los gastos son cristalizados de manera eficiente, para ello se hace uso de la planeación y de la racionalización de la actuación del Estado. Es por esta 
razón que Aguilar (1996) manifestó que en lo económico se pasó de un modelo intervencionista a un modelo de desarrollo neoliberal.

En definitiva, el diseño y la implementación de políticas públicas efectivas representan los instrumentos socialmente disponibles, en que se gestiona el uso estratégico de los recursos para aliviar problemas nacionales, lograr beneficios convergentes a todos los grupos que están involucrados y asegurando de esta manera una prestación económicamente eficiente, socialmente equitativa y ambientalmente sustentable.

\section{Desarrollo sostenible rural}

El uso sostenible del agua consiste en utilizar el recurso hídrico para diferentes actividades que tienen resultados productivos y de bienestar social, sin que se produzca una degradación de las dinámicas naturales que permiten su disponibilidad en cantidad y calidad; es decir, sin degradar la cuenca hidrográfica.

\section{MATERIALES Y MÉTODOS}

Esta investigación se realizó utilizando una metodología cualitativa, documental, con una revisión de documentos y trabajo de los autores más significativos que han hecho estudios sobre zonas rurales, correlacionando los aspectos más importantes que son el denominador común que cada autor considera necesario para el desarrollo rural. Por lo que esta investigación tiene como objetivo analizar la importancia de las políticas públicas en el desarrollo rural de la parroquia de Manglaralto y en el manejo sostenible del acuífero costero.

El planteamiento de políticas públicas adecuadas, que permitan la aplicación directa a los problemas que ya están inmersos las comunas que se abastecen del acuífero. Se pretende a través de las ciencias de las políticas formular una propuesta considerando las características geográficas y culturales de la zona.

En esta investigación se busca examinar el efecto que tendría el planteamiento de unas políticas públicas adecuadas en el desarrollo sostenible del acuífero. Por otro lado, esta investigación busca describir la forma de organizarse de los comuneros y su accionar frente a la junta de agua potable frente a la necesidad de abastecerse de este líquido vital.

\section{RESULTADOS Y DISCUSIÓN}

Los acuíferos son reservorios naturales que se recargan naturalmente, por lo que es indispensable que estos no sean sobreexplotados ya que atraerían problemas, convirtiendo a esta fuente de agua vulnerable, reduciendo las reservas de agua dulce.

Por otro lado, las políticas públicas partiendo del concepto de que es una acción o un conjunto de acciones provenientes de la decisión de las autoridades legítimas vislumbra un mecanismo para resolver situaciones problemáticas entre ellas, el abastecimiento de agua potable y la conservación de los acuíferos naturales.

Por lo que, es indispensable que se adapten ajustes en las políticas públicas de agua en zonas rurales, para direccionar el manejo sostenible del acuífero costero en la Parroquia de Manglaralto.

Es importante determinar cómo menciona el marco regulatorio en el Ecuador, de las políticas pública de agua en zonas rurales, en la formulación de los lineamientos de las políticas públicas, y como la identificación de los problemas en cuanto al acceso al agua y sostenibilidad del acuífero en la parroquia de Manglaralto, en la formulación de los lineamientos de las políticas públicas.

También se requiere analizar la incidencia que tendría un modelo participativo por parte de la comunidad local, en la formulación de los lineamientos de las políticas públicas. 
Este trabajo de investigación se basa en la necesidad de buscar el desarrollo sostenido del acuífero, por lo que el resultado de esta investigación permitirá plantear las políticas públicas más adecuadas de acuerdo a la zona y a las características naturales y la cultura de las personas para el desarrollo sostenible del acuífero y así lograr un impacto positivo en la comunidad, como para los ecosistemas que depende del acuífero.

Debido a que el agua extraída del acuífero natural permite cubrir algunas de las necesidades básicas de los comuneros es primordial proponer las políticas públicas más idóneas que permitan su preservación y conservación de este acuífero, que, de otro modo, si faltare este recurso vital, las personas se verían seriamente afectadas en sus actividades más básicas y también en las actividades económicas que se realiza con el agua como lo es la agricultura y el turismo.

Partiendo del concepto de que las políticas públicas es una acción o un conjunto de acciones provenientes de la decisión de las autoridades legítimas cuyo objetivo es resolver situaciones problemáticas, aplicadas considerando el contexto del lugar, las características naturales, las necesidades sociales, económicas de los pobladores producirían una transformación socioeconómica en el sector y se propiciaría el desarrollo sostenido del acuífero, fuente fundamental para el suministro del agua en la zona. Como una política pública debidamente preconcebidas propiciarían un desarrollo sostenido para la comunidad, así como para la preservación de este acuífero natural y como las comunas, reconocida su forma de asociación en la constitución, como una práctica ancestral, a través de su actuación favorecerían a sus comuneros, así como a la preservación del acuífero y con ello los ecosistemas inmersos.

Para (López Vera, 2002) la introducción de estrategias de gestión de calidad de las aguas en las agendas y el ciclo político, toma mucho tiempo por considerarse un proceso largo y si se incluye es como resultado de presiones tanto sociales como de los medios de comunicación, conside ra que las estrategias enfocadas a la protección de la captación de agua, así como el establecimiento de una cartografía de vulnerabilidad a la contaminación y de redes de control, mediante pozos y piezómetros de observación y muestreo son insuficientes, se requiere de otras estrategias que involucren programas más amplios y abarquen el manejo de contaminantes control de vertidos en origen y la ordenación del territorio.

Para (López Vera, 2002) es enfático en sostener que a pesar de que en los foros internacionales con la temática del cuidado y protección de los recursos hídricos se pone sobre aviso de los latentes peligros de contaminación de las aguas subterráneas y sobre la urgente necesidad de que los gobiernos de los países, adopten medidas inmediatas de protección de los acuíferos tanto en calidad como en cantidad, al ser fuentes de abastecimiento de agua potable y muy a pesar de que los gobiernos suelen adherirse firmando estos principios, aún existe el vacío de la inclusión de medidas concretas en las políticas nacionales, siendo muy irregular y a expensas de que existan o no grupos que presionen para que se las incluyan en las agendas políticas.

Considerando lo que se está haciendo en otras partes para la gestión de la calidad del agua en la unión Europea, las estrategias que propone (López Vera, 2002), van más allá de las medidas comunes, y sostiene que deben incluirse programas con un plazo de cuatro años, como el análisis de la demarcación hidrográfica, un estudio de las consecuencias de la actividad humana en la calidad y cantidad de las aguas subterráneas, registrar las zonas declaradas de protección esencial para la preservación de los acuíferos y de los hábitats que se mantienen de ellas.

Así mismo, la participación de la comunidad en la gestión del agua, permite que sean actores activos, comprometidos e involucrados en los lineamientos de la agenda política, donde expongan sus necesidades inmediatas. Por lo tanto, en el paso de diseñar las políticas públicas, permita este proceso la participación activa de la comunidad y que el cuidado de este recurso hídrico sea compartido con los pobladores y ellos sea a su vez los primeros que monitoreen y controlen eficientemente el uso y la aplicación de este recurso. 


\section{CONCLUSIÓN}

Es necesario que se incluyan en las agendas políticas, las medidas de protección de las aguas subterráneas, considerando que como base jurídica constan los compromisos adquiridos por el gobierno referente a este tema y a todas las recomendaciones que han dado los organismos internacionales, no esperar que alguna medida se tome como emergencia cuando puede ser ya muy tarde, estas medidas deben ir más allá del accionar del gobierno nacional, sino que también se traslade la responsabilidad a los gobiernos seccionales y a todos los organismos públicos que estén inmersos en la gestión de las aguas subterráneas.

Las políticas públicas a nivel país, concernientes a la gestión del agua, están enfocadas a las grandes ciudades y se basa en proyectos de inversión de grandes obras de infraestructura hidráulica, los gobiernos autónomos descentralizados, siguen teniendo serios problemas y con muchas limitaciones para atender cada una de las demandas por acceso al agua y en visto a estas limitaciones, las comunidades rurales se han hecho presente, por iniciativa propia se han organizado y creado las Juntas de Agua. Es por ello, que su participación es indispensable y su aporte es valioso para obtener el bien común a todos los miembros de sus comunas.

\section{REFERENCIAS}

Adshead, M. (julio de 1996). Beyond Clientelism Agricultural Networks in Ireland and the EU. West European Politics, 19(3), 583-608.

Almond, G., \& Powell, B. (1978). Política Comparada. Buenos Aires: Paidós.

Bautista C., N. P. (s.f.). Proceso de la investigación cualitativa: epistemología, metodología y aplicaciones. Bogotá: El Manual Moderno Colombia.

Bernal, C. (2010). Metodología de la investigación. Colombia: Pearson.

BID, \& ONU-HABITAT. (2011). Acceso al agua y al saneamiento para todos y el derecho.

Cerda, H. (1998). Los elementos de la investigación. Bogota: El Búho.

Desarrollo, S. N. (2017). Plan Nacional de Desarrollo 2017 - 2021 Toda una Vida. Quito .

Dunleavy, P. (Agosto de 1981). Professions and Policy Change. Public Administration Bulletin, 36, 3-16.

Dunleavy, P., \& W, N. D. (1981). Public Policy Analysis. Englewood Cliffs: Prentice Hall.

Dye, T. R. (1992). Understanding public policy. Nueva Jersey: Prentice Hall.

Ecuador, R. d. (2014). Ley Orgánica de recursos hídricos, usos y aprovechamiento del agua.

Edwards, G. C., \& Sharkansky, I. (1978). The Policy Predicament. San Francisco: W.H. Freeman \& Co.

Fàbregues Feijóo, S., Meneses Naranjo, J., \& Rodríguez Gómez, D. (2016). Técnicas de investigación social y educativa. Barcelona: Editorial UOC. Obtenido de http://puceftp.puce.edu.ec

Flora, P., \& Heidenheimer. (1981). The Development of Welfare States in Europe and America. New Brunswick, N.J.: Transaction Books.

Friedman, J. (1991). Planificación en el ámbito público. Madrid: MAP. 
Fuentelsaz Gallego, C., Icart Isern, M. T., \& Pulpón Segura, A. M. (2006). Elaboración y presentación de un proyecto de investigación y una tesina. Barcelona: Edicions Universitat Barcelona,.

Gallardo Vázquez, P., \& Camacho Herrera, J. M. (2008). La investigación participativa y su aplicación en el campo social y educativo. Sevilla: Wanceulen Editorial.

Goodin, R., \& Klingemann, H. D. (2001). Nuevo Manual de Ciencia Política. Madrid: Istmo.

Hernández, R. F., \& Baptista, P. (2010). Fundamentos de metodología de la investigación . México: McGraw Hill.

INEC. (2010). Censo de Población y Vivienda.

Kitzinger, J. (1995). Introducing focus groups, Sociology of Health. Education and debate Qualitative Research(311), 299-302. Obtenido de www.bmj.com/cgi/content/full/311/7000/299

Lasswell, H. (1996). La concepción emergente de las ciencias. En L. F. Villanueva, El estudio de las políticas (págs. 105-118). México D.F.: Miguel Ángel Porrua.

Lindblom, C. E., \& Cohen, D. (1979). Usable Knowledge:Social Science and Social Problem Solving. New Haven: Yale University Press.

LORH. (2014). LEY ORGÁNICA DE RECURSOS HÍDRICOS,USOS Y APROVECHAMIENTO DEL AGUA.

Losada Trabada, A. (2003). Entre la ciencia política básica y la ciencia política aplicada; de la política a las políticas, del análisis a la gestión. RIPS- Revista de Investigaciones Políticas, 2 (1-2), 63-81.

Majone, G. (1989). Evidence, Argument \& Persuasion. New Haven: Yale University Press.

Majone, G. (2001). Políticas públicas y administración:. En R. G. Klingemann., Nuevo Manual de Ciencia Política (págs. 887-913). Madrid: Istmo.

Many, I., \& Thoenig, J.-C. (1992). Las políticas públicas. Barcelona: Ariel, S.A.

Medellín, P. (2004). La política de las políticas públicas:propuestas teóricas y metodológicas para el estudio de las políticas públicasen países de frágil institucionalidad. Serie Políticas Sociales $\mathrm{N}^{\circ}$ 93, CEPAL. Santiago de Chile.

Molina, I., \& Delgado, S. (2001). Conceptos fundamentales de Ciencias Política. Madrid: Alianza Editorial.

Monnier, E. (1995). Evaluación de la acción de los poderes públicos. Madrid: Instituto de Estudios Fiscales.

Muller, P. (1998). Génesis y fundamentos del análisis de políticas. Innovar. Revista de ciencias administrativas y sociales(11), 99-109.

Muller, P. (diciembre de 2002). Las políticas públicas. Administración y Desarrollo(38), 116-119.

Muller, P. (2006). Las políticas públicas. Bogotá: Editorial Universidad Externado de Colombia.

Muller, P. (2007). Prefacio. En J. I. Cuervo, Ensayos sobre políticas públicas (págs. 11-13). Bogotá: Editorial Universidad Externado de Colombia.

Myers, G. (. (1998). Language in Society. Displaying opinions: topics and disagreement in focus groups, 27(1), 85-111. 
Nelson, B. (2001). Políticas públicas y administración: una visión general . En R. G. Klingemann, Nuevo Manual de Ciencia Política (págs. 795-860). Madrid: Istmo.

ONU. (2010). La Observación General núm. 15.

PDyOY. (2014). Plan de Desarrollo y Ordenamiento Territorial 2014-2019.

REpublicadelEcuador. (2008). Constitución de la República del Ecuador. Quito-Ecuador.

Roth Deubel, A.-N. (2002). Políticas públicas. Formulación,implementación y evaluación. Bogotá: Ediciones Aurora.

Salazar Vargas, C. (1995). Las políticas públicas. Serie Colección Profesores.

Salazar Vargas, C. (1995b). Las políticas públicas: nueva perspectiva de análisis. Ciencia Política, $33,59-80$.

Salkind, N. (1998). Método de Investigación. México: Prentice-Hall.

SENPLADES. (2017). Plan Nacional de Desarrollo 2017-2021. Toda una Vida. Quito-Ecuador .

Subirats, J. (1989). Análisis de políticas públicas y eficacia de la administración. Madrid: Ministerio para las administraciones públicas.

Subirats, J. (1992). Un Problema de Estilo: La Formación de Políticas Públicas en España. Madrid: Centro de Estudios Constitucionales.

Subirats, J. (1999). ¿existe sociedad civil en España? Responsabilidades colectivas y valores públicos. Madrid: Fundación Encuentro.

Subirats, J., \& Gomà., R. (1998). Políticas públicas en España. Contenidos, redes de actores y niveles de gobierno. Barcelona: Ariel.

Thoening, J. C. (1885). L'analyse des politiques publiques. En M. Grawitz, \& J. Leca, Traité de science politique. París: PUF.

Yuni, J., \& Urbano, C. (2006). Técnicas para Investigar. Recursos metodológicos para la. Córdoba: Editorial Brujas. 